九州大学学術情報リポジトリ

Kyushu University Institutional Repository

Estimating the Coverage Performance of a

Wireless Sensor Network Considering Boundary

Effects in the Presence of Sensor Failure

Mini

Department of Mathematics, Chandigarh University

Pal, Ashok

Department of Mathematics, Chandigarh University

https://doi.org/10.5109/4491652

出版情報 : Evergreen. 8 (3)，pp.601-609，2021-09. 九州大学グリーンテクノロジー研究教育センター バージョン:

権利関係 : 


\title{
Estimating the Coverage Performance of a Wireless Senso $r$ Network Considering Boundary Effects in the Presence of Sensor Failure
}

\author{
Mini $^{1,2 *}$, Ashok $\mathrm{Pal}^{1)}$ \\ ${ }^{1}$ Department of Mathematics, Chandigarh University,Mohali, Punjab, India \\ ${ }^{2}$ S. A. Jain (PG) College, Ambala City, Haryana, India \\ *Author to whom correspondence should be addressed: \\ E-mail:miniarora49@gmail.com
}

(Received March 1, 2021; Revised June 17, 2021; accepted July 25, 2021).

\begin{abstract}
Coverage is one of the most critical performance metric of wireless sensor networks (WSNs) because it shows how well a region of interest (RoI) is being monitored by the deployed network. In general, the RoI is either circular or rectangular in shape which have boundary regions. Sensor nodes (SNs) deployed in these regions suffer boundary effects (BEs), i.e., the useful coverage area of an SN deployed near the boundary regions is less as compared to the SNs deployed in the middle of the RoI. It is imperative to consider these BEs while evaluating the performance of WSNs because analytical results derived for large networks are not valid for finite networks. In addition, SNs of a deployed WSN are prone to failure due to a large number of factors such as battery drainage, high temperature, and other environmental conditions. Earlier researchers have ignored the impact of BEs in the presence of sensor failure while evaluating the coverage performance of WSNs. In this work, we derive an analytical model by considering BEs and sensor failure to achieve a closed form expression for the $k$-coverage performance of a WSN deployed in a rectangular RoI. Further, we analyze the influence of various network parameters such as number of SNs, sensing range, and sensor failure rate on the $k$-coverage performance of the network. The results obtained using the proposed model show a good match with simulations outcomes with Root Mean Square Error (RMSE) no more than 0.03 , thus, validating our model. For $r_{s}=80 \mathrm{~m}$ and $N=100$, 1-coverage probabilities are found to be $0.9874,0.9313$ and 0.8069 for $k=1,2$ and 3 respectively showing that the $k$-coverage probability deriorates with the increase in the value of $k$.
\end{abstract}

Keywords: wireless sensor networks; binary sensing model; $k$-coverage probability; sensor failure rate; boundary effects.

\section{Introduction}

This is the era of technological advancements and innovations in the field of wireless communication. As a result, devices with wireless communication abilities such as SNs can be produced at a large scale in different shapes and sizes. These wireless SNs are deployed to create different types of wireless networks such as WSNs, wireless multihop networks, wireless ad hoc networks and so on ${ }^{1,2)}$. The popularity of these networks is due to the reason that they can be formed on the fly easily and operate in a decentralized fashion without requiring any fixed infrastructure such as base station ${ }^{3}$. As a result, they are being deployed for a large number of military and civilian applications such as border surveillance, enemy tracking andreconnaissance, precision agriculture, landslide monitoring, wildlife monitoring, seismic activity monitoring, industrial automation, ubiquitous and pervasive computing, health industry and so on ${ }^{4,5,6,7,8,9)}$.
Coverage and connectivity are two essential performance metrics of WSNs because coverage determines how well an RoI is being monitored by the deployed network and the connectivity allows the network nodes to send their sensed data to the sink node ${ }^{10,11,12,13)}$. As per the application requirements, the coverage metric of a network can obtained in terms of point coverage, partial area coverage, full area coverage, and $k$-coverage. Some critical applications such as military surveillance require consistent monitoring of the region and cannot bear coverage holes caused by the nodes failure and other environmental conditions, thus, they demand $k$-coverage of the network to have a network which is robust against nodes failure.

It has been observed that analytical models developed for large networks cannot be applied for finite networks because of BEs, i.e., the useful coverage area of a node deployed in boundary regions is less than the useful 
coverage area of the node in inner region. It is due to the reason that BEs distort the linearity and dependencies between the network parameters and variables which in tern lead to the dynamic behavior of network ${ }^{14,15}$. There exits a plethora of literature addressing the coverage issue in WSNs ${ }^{16,17,18,19,20,21,22,23,24)}$. Real time monitoring of mines and tunnels is crucial to avoid anycatastrophe. Thus, a WSN capable of detecting underground tunnels and open-pit mines damage in real times is proposed in refernce $^{16)}$. In literature ${ }^{17)}$, the authors have proposed a distributed border surveillance system for a WSN deployed in a circular and rectangular RoI. They have obtained the number of barrier paths using binary and lognormal shadowing sensing range model and observed that the log-normal shadowing sensing range model out performs binary sensing range model. The work presented in literature ${ }^{18)}$ studied the weak $k$-barrier coverage for underwater WSNs and proposed an efficient algorithm to obtain two-dimensional weak $k$-barrier coverage from three-dimensional weak $k$-barrier coverage. Authors in literature $^{19)}$ have rendered an analytical model to investigate the $k$-barrier coverage probability for the intrusion detection in WSN. Further, they have examined the impact of sensing range, node density, and sensor speed on the intrusion detection probability. The authors $i^{20)}$ have rendered a method to track and detect the boundary of a consistent object using WSNs. Further, an effective probabilistic sensing range model for the intrusion detection in mobile sensor networks has been proposed in literature ${ }^{21)}$. The authors have observed that coverage performance of the network improves with the increase in the speed of sensor nodes and becomes equal to the intruder detection time considering Boolean sensing range model. The wireless channel environment have a large number of natural phenomena taking place in it such as interference, multipath fading, scattering, reflection and refraction. The work presented in literatures ${ }^{22,23)}$ studied the impact of wireless channel environment on the coverage metric of WSNs and found that interference and multipath fading have severely deteriorating influence on the cov- erage metric of the deployed network. Nowadays, artificial intelligence and machine learning algorithms are being applied in the domain of $\left.\mathrm{WSNs}^{24}, 25,26\right)$. In literature $^{25}$, the authors have proposed three machine learning algorithms to predict the average localization error in the position of SNs at the time of deployment. The authors found that their proposed algorithms are very efficient with Root Mean Square Error (RMSE = 0.147m).

Another work presented in literature ${ }^{26)}$ employed proposed a Gaussian regression based method to estimate the $k$-barrier coverage probability for the intrusion detection in WSNs. The authors have also investigated the impact of sensing range, number of nodes, sensor to intruder speed ration, and static to mobile sensor ration on the $k$-barrier coverage probability of the network.

Although, the above discussed work are very effective and can be implemented for various civil and military applications, but, they have major drawback of ignoring BEs and sensor nodes failure on the coverage performance of the networks. In this work, we have formulated an analytical model by considering BEs and sensor nodes failure to estimate the k coverage probability of a WSN deployed in a rectangular RoI. Following are the major contributions of the paper in hand:

1.An analytical model comprising of boundary effects and sensor nodes failure to estimate the $\mathrm{k}$ coverage probability of a wireless sensor network deployed in a rectangular region.

2.Assessing the influence of number of sensor nodes, sensing range, and sensor node failure rate on thek coverage probability of the deployed network.

Rest of the paper is arranged as follows: System model comprising of node distribution model, sensing range model and nodes' failure rate is discussed in Section 2. In Section 3, we have calculated the useful coverage area of nodes in boundary and non-boundary regions. Then, the analytical formulation of $k$-barrier coverage probability is presented in Section 4. The analytical and simulation results are discussed in Section5. Finally, the paper is concluded in Section 6.

\section{System Model}

We have considered a rectangular RoI having length $I$ and width $w$ meters. A finite number $(N)$ of identical immobile SNs are deployed uniformly and independently inside the RoI. Each SNs is assumed to have identical computational, sensing and transmission capabilities. Therefore, the sensing range of each node is assumed to be $r_{s}$ meters and the coverage area is $\pi r_{s}^{2}$ while neglecting the BEs. An arbitrary point inside the RoI is said to be covered if it lies within the sensing range of at least one node. Furthermore, the RoI is said to be covered by the deployed network if every point inside the RoI lies within the sensing range of at least one node. In addition, the system model comprises of a node distribution model, sensing range model and nodes failure rate which are explained below:

\subsection{Node Distribution Model}

In a realisticscenarios such as forests fire, battlefields, enemy territories and so forth, the deterministic deployment of SNs is not possible. This is where the uniform distribution is very handy and can be obtained easily with the help of low flying aeroplanes. According to this model, the SNs are spread uniformly and independently inside a given RoI, i.e., the probability of lying on any point inside the rectangular RoI is same for every $\mathrm{SN}$ and is independent of the position of other SNs.

\subsection{Sensing Range Model}

There exists a variety of sensing range models such as binary sensing range model, Elfes' sensing rage model, 
shadowing sensing range model, two ray ground propagation model, and so on ${ }^{4,27)}$. To keep it mathematically tractable, we consider the most widely used binary sensing range model. According to this model, a random point inside the RoI will be covered by an $\mathrm{SN}$ if and only if it lies within the sensing range of the SN. Mathematically, it can be represented by Eq. 1 .

$$
P_{c o v}(r)=\left\{\begin{array}{l}
1, r \leq r_{s} \\
0, r>r_{s}
\end{array}\right.
$$

\subsection{Node failure probability}

An $\mathrm{SN}$ is an electronic device whose lifetime may follow an exponential, a normal or a weibull distribution model. These distribution models have been used to model the lifetime of SNs in many research articles ${ }^{27}$. All these models provide the lifetime of SNs in terms of probability, i.e., the probability that an $\mathrm{SN}$ will not fail before a given period of time. Therefore, to avoid the mathematical complexities of these model, we have considered the SN failure probability representing chances of failure for a given $\mathrm{SN}$.

\section{Useful Coverage Area of an SN}

Here, we compute the useful coverage area of an SN lying inside a rectangular RoI. For this reason, the whole rectangular RoI is divided into different boundary and non-boundary regions as shown in Fig. 1. Then, we compute the average useful coverage area of an SN lying in these boundary and non-boundary regions, which in turn is used to compute the $k$-coverage probability of the deployed WSN.

\subsection{Useful coverage area of an $\mathrm{SN}$ without $\mathrm{BEs}$}

For a rectangular RoI where a given number of SNs $N$ are distributed uniformly and independently. The probability that the an $\mathrm{SN}$ will lie at a given point $P(x, y)$ inside the RoI is $P_{I}=(1 / l w)$. If the dimensions of the rectangular region are very high, then the BEs diminish and have negligible impact on the network coverage. Therefore, the useful coverage area of an SN will be a circular disk of radius $r_{s}$, i.e., $A_{1}=\pi r_{s}^{2}$. Therefore, the average probability that an arbitrary point inside the RoI will be covered by an SN is computed using Eq. 2 .

$$
E(C)=P_{I} A_{I}
$$

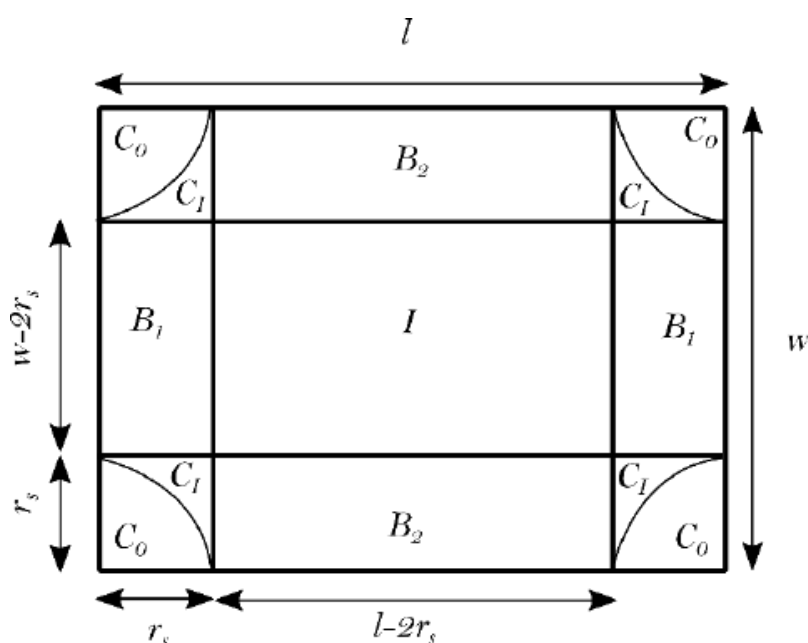

Fig.1:Boundary $\left(B_{1}, B_{2}, C\right)$ and non-boundary $(I)$ regions of a rectangular region.

\subsection{Useful coverage area of an $\mathrm{SN}$ with BEs}

It is an established fact that the BEs have a deteriorating impact on the coverage and connectivity performance of $\mathrm{WSNs}^{3,28)}$. For a given sensing range of SNs, the whole rectangular RoI can be partitioned into an inner region $(I)$, lateral boundary regions $B_{1}$, and $B_{2}$, and corner regions $C$ as shown in Fig. 1 . It is noteworthy that the number of boundary regions for each $B_{1}$ and $B_{2}$ are two. Furthermore, each corner region is divided into two subregions, viz., inner corner $C_{I}$ and outer corner $C_{O}$ as shown in Fig. 1. The useful coverage area an arbitrary SN lying in these regions is calculated as follows:

Inner region $(I)$ : In this region, a node lying at a point $P_{I}(x, y)$ does not suffer any kind of BEs as shown in Fig. 2 , as a result, the useful coverage area of node in region $I$ is a circular disk of radius $r_{s}$ and is given by Eq. 3

$$
A_{I}=\pi r_{s}^{2}
$$

and the probability that an arbitrary sensor node will lie in inner region $I$ of the rectangular region is given by Eq. 4

$$
P_{I}=\frac{\left(l-2 r_{s}\right) \times\left(w-2 r_{s}\right)}{l \times w}
$$

Lateral boundary region $\left(B_{1}\right)$ : Let us assume that an SN is lying at a point $P_{B 1}(x, y)$ in lateral boundary region $B_{1}$. In this region, the useful coverage area of the SN will be less than $\pi r_{s}^{2}$ because some of thecoverage area of the SN lies outside the RoI as shown in Fig. 2. 


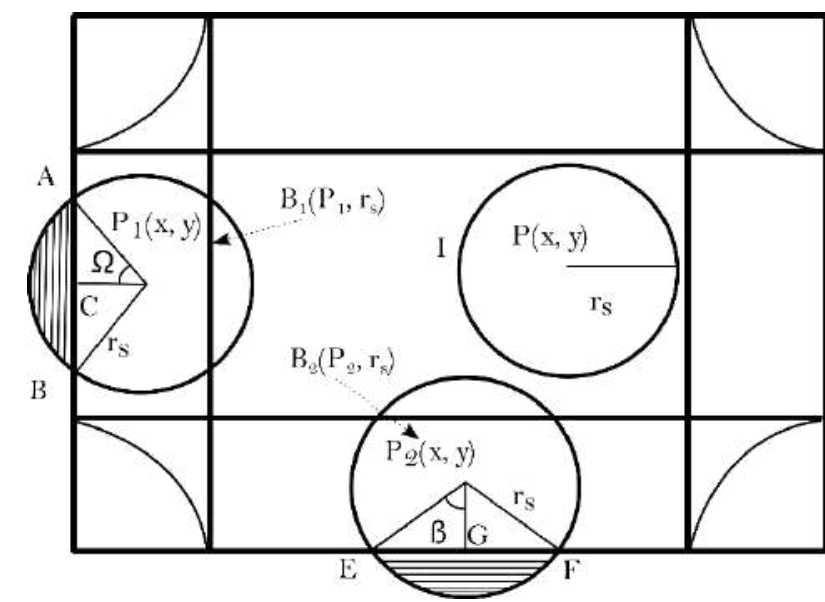

Fig.2: Useful coverage area of a node in region $I, B_{1}$, and $B_{2}$.

Therefore, the useful coverage area of theSN in region $\left(B_{1}\right)$ is obtained by subtracting the circular area lying outside the RoI from $\pi r_{s}^{2}$. The circular region lying outside the region can be obtained by subtracting the area of the triangular portion from the circular arc of radius $r_{s}$. The average useful coverage area of a node in region $\left(B_{1}\right)$ and $\left(B_{2}\right)$ are equal ${ }^{12}$ and be computed using Eq. 5 .

$$
A_{\text {lat }}=A_{B_{1}}=A_{B_{2}}=\left(\pi-\frac{2}{3}\right) r_{s}^{2}
$$

and the probability that an arbitrary sensor node will lie in a lateral boundary regions is given by Eq. 6 .

$$
P_{l a t}=\frac{2 r_{s} \times\left(l-2 r_{s}\right)+2 r_{s} \times\left(w-2 r_{s}\right)}{l \times w}
$$

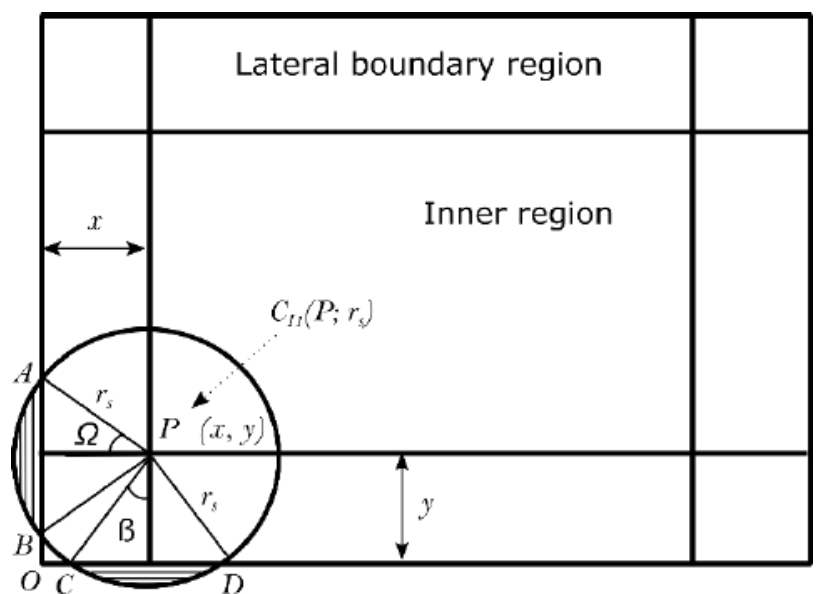

Fig.3:Useful coverage area of a node in inner corner region $C_{I}$

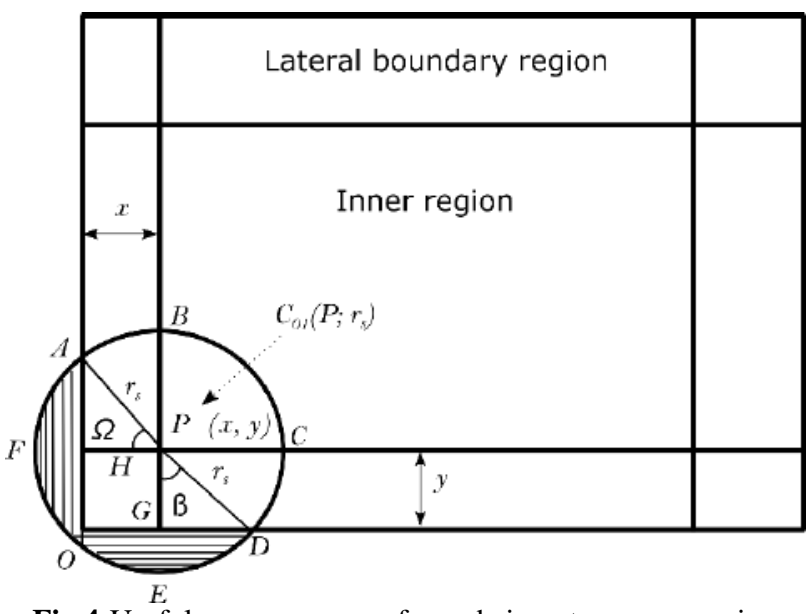

Fig.4:Useful coverage area of a node in outer corner region Co.

Inner corner region $\left(C_{I}\right)$ : Let us assume that an $\mathrm{SN}$ is lying at a point $P_{C I}(x, y)$ in inner corner region $C_{I}$ of the rectangular RoI. Here, it is important to notice that the coverage area of the $\mathrm{SN}$ is affected by two lateral boundaries $B_{1}$ and $B_{2}$ as shown in Fig. 3. Therefore, the useful coverage area of the $\mathrm{SN}$ in inner corner region $\left(C_{I}\right)$ can be computed by subtracting the area of two circular segments formed outside $B_{1}$ and $B_{2}$ from $\pi r_{s}^{2}$. Thus, the average useful coverage area of an $\mathrm{SN}$ in inner corner region $C_{I}$ is given by Eq. $7\left(\right.$ refer $\left.^{12}\right)$.

$$
\mathrm{A}_{\mathrm{C}_{\mathrm{I}}}=\left(\frac{\pi^{2}+1}{2 \pi}\right) \mathrm{r}_{\mathrm{s}}^{2}
$$

Outer corner region $\left(C_{O}\right)$ : Let us assume that an $\mathrm{SN}$ is lying at a point $P_{C O}(x, y)$ in outer corner region $C_{O}$ of the rectangular RoI. Here, the coverage area of the $\mathrm{SN}$ is lying outside lateral boundary regions $B_{1}, B_{2}$ and vertex $V_{I}$ as shown in Fig. 4. Therefore, the average useful coverage area of the $\mathrm{SN}$ in outer corner region $\left(C_{O}\right)$ can be calculated by subtracting the area of two circular segments formed outside $\mathrm{B}_{1}$ and $\mathrm{B}_{2}$ and the area lying outside vertex $V_{I}$ from $\pi r_{S}^{2}$ and is given by Eq. 8 .

$$
A_{C_{O}}=\frac{4 r_{s}^{2}\left(\pi-\frac{4}{3}-\frac{\pi^{2}}{8}\right)}{(4-\pi)}
$$

The expected useful coverage area of an SN lying in a corner region is obtained by averaging over the entire circular region and is given by Eq. 9

$$
A_{C}=\left(\pi-\frac{29}{24}\right) r_{s}^{2}
$$

and the probability that an arbitrary SN will be lying in a corner region can be computed using Eq. 10.

$$
P_{C}=\frac{4 r_{s}^{2}}{l \times w}
$$




\section{Coverage Probability}

Let $P_{i}$ and $A_{i}$ denote the probability that an arbitrary $\mathrm{SN}$ is positioned in region $i$ and the useful coverage area of the $\mathrm{SN}$ in region $i$ respectively, where $i \in\left[I, B_{1}\right.$, $\left.B_{2}, C\right]$.Therefore, the expected coverage of an arbitrary SN can be calculated using Eq. 11

$$
E(C)=\frac{P_{I} A_{I}+P_{l a t} A_{l a t}+P_{C} A_{C}}{l \times w}
$$

If $P_{f}$ denotes the failure probability of an $\mathrm{SN}$, then the probability $\left(P_{c}\right)$ that a random point within the RoI will be covered by an arbitrary SN is given by Eq. 12

$$
P_{C}=E(C)\left(1-P_{f}\right)
$$

Then, the probability that the target would not be covered by any SN of the network can be computed by Eq. 13

$$
P_{N C}=\left\{1-E(C)\left(1-P_{f}\right)\right\}^{N}
$$

Finally, the probability that the target would be covered by at least one SN of the network, i.e., 1-coverage of the network can be calculated using Eq. 14

$$
\begin{gathered}
=1-P_{N C} \\
=1-\left\{1-E(C)\left(1-P_{f}\right)\right\}^{N}
\end{gathered}
$$

In order to achieve a robust network against node failure, we estimate the $k$-coverage probability of the network. The probability that an arbitrary point inside the given RoI will be covered by exactly $k$ distinct SNs can be computed using Eq. 15.

$$
P_{k}=\left(\begin{array}{l}
N \\
k
\end{array}\right)\left(P_{C}\right)^{k}\left(1-P_{C}\right)^{N-k}
$$

and the probability that an arbitrary point inside the RoI will be covered by at least $k$ distinct SNs is given by Eq. 16

$$
\begin{gathered}
C_{k}=1-\sum_{K=0}^{k-1} P_{k} \\
=1-\sum_{K=0}^{k-1}\left(\begin{array}{l}
N \\
k
\end{array}\right)\left(P_{C}\right)^{k}\left(1-P_{C}\right)^{N-k}
\end{gathered}
$$

\section{Results and Discussion}

In this section, we present the analytical and simulation results. The analytical results for $k$-coverage probability are obtained by putting Eq. 2 (without BEs) and Eq. 11 (with BEs) in Eq. 16.

Table 1. Simulation parameters
\begin{tabular}{|l|l|}
\hline Parameter & Value(s) \\
\hline Dimension of the rectangular region $(l, w)$ & $\begin{array}{l}l=500 \mathrm{~m}, \\
w=400 \mathrm{~m}\end{array}$ \\
\hline Number of sensor nodes $(N)$ & $5-200$ \\
\hline Sensingrange $\left(r_{s}\right)$ & $0-120 \mathrm{~m}$ \\
\hline Sensor failure rate $\left(P_{f}\right)$ & $0-0.8$ \\
\hline Required $k$ & $1-3$ \\
\hline
\end{tabular}

\subsection{Simulation Setup}

Simulation results are obtained through the extensive simulations on MATLAB 2018b using the parameters given in Table 1 . The entire rectangular region is divided into many alike squares of area $1 \mathrm{~m} \times 1 \mathrm{~m}$. For each iteration, it is checked whether a given square regions falls within the sensing range of an SN or not without changing the topology. Then, the square is considered to be covered by the network if and only if its center is detected by at least one SN. Then, the network coverage is achieved by diving the number of covered squares by the total number of squares in entire network. Eventually, the single $k$ coverage outcome is obtained by taking the mean of 10000 iterations for a randomly spread network.

\subsection{Influence of sensing range on network coverage}

In this section, we assess the influence of sensing range of SNs on the $k$-coverage metric of the network at different values of $P_{f}$. We have observed that the $k$-coverage probability of the network improves with the increase in the sensing range of SNs at a given value of $P_{f}$ as shown in Fig. 5, and Fig. 6. For instance, the 1-coverage probabilities of the network are found to be 0.7004 , 0.8784 and 0.9608 when the sensing ranges are $30 \mathrm{~m}, 40$ $\mathrm{m}$ and $50 \mathrm{~m}$ respectively at $P_{f}=0.4$ (refer Fig. 5). This is because of the reason that sensors with large sensing range can cover points lying at large distances inside the RoI. As a results, the number of squares covered increases which in tern improves the $k$-coverage probability. However, the $k$-coverage probability deteriorates with the increase in the value of sensor failure rate $\left(P_{f}\right)$ at a given value of SNs $(N)$ and sensing range $\left(r_{s}\right)$ as indicated in Fig 5, and Fig 6 For instance, at $r_{s}=50 \mathrm{~m}$ and $N=150$, 1-coverage probabilities are found to be $0.9950,0.9867,0.9608$, 0.8835 and 0.6576 when the values of $P_{f}$ are $0,0.2,0.4$, 0.6 and 0.8 respectively. This is caused by the fact that at higher values of $P_{f}$, the expected number failed SNs will be high, thus a less number of active SNs will cover a small fraction of the squares which in tern will decrease the 1-coverage probability. We have observed the similar trends while increase the value of $k$ for $k$-coverage probability as shown in Fig 6. The $k$-coverage probability decreases with the increase in the value of $k$ because a large value of $k$ demands that a given point inside the RoI 


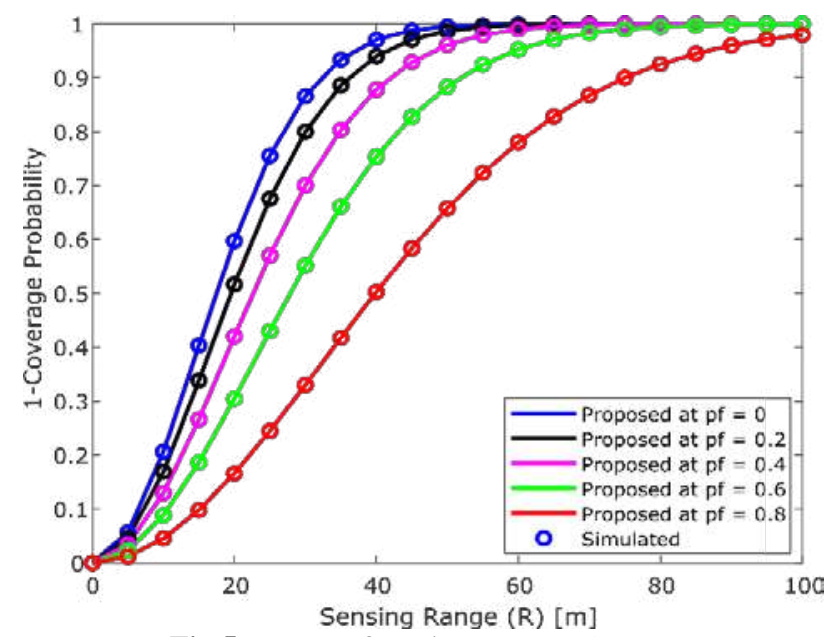

Fig.5:Impactofsensingrangeon1coverageprobabilityatdifferentvaluesofnodefailurerate $\left(\boldsymbol{P}_{f}\right.$ )and $N=150$.

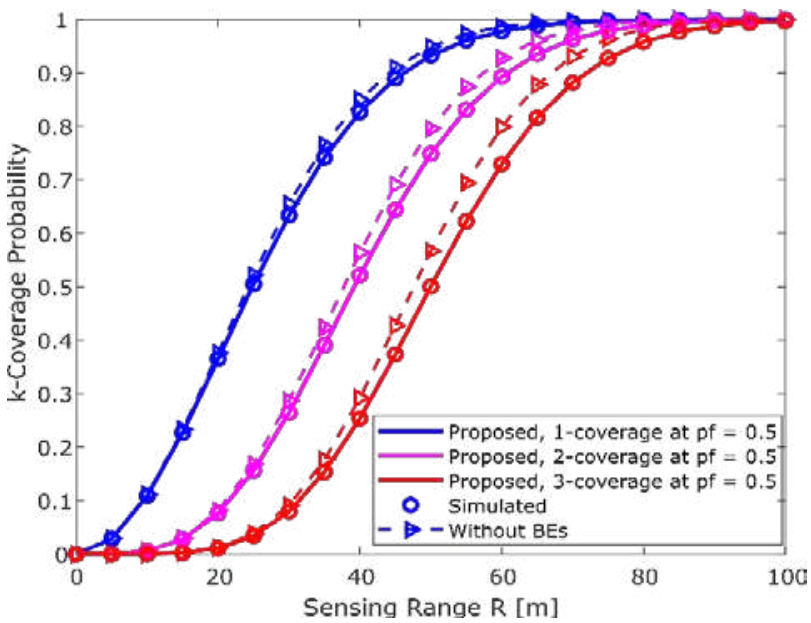

Fig.6:Impact of sensing range on $k$-coverage probability when node failure rate $\left(P_{f}=0.5\right)$ and $N=$ 150.

must be covered by at least $k$ distinct $\mathrm{SNs}$ at a given value of $N$ and $r_{s}$. In addition, Fig. 6 also shows that there is a significant difference between the results ignoring and incorporating BEs. The simulation results match with the results obtained through the proposed model, thus confirming our model.Here, we investigate the influence of number of SNs on the $k$-coverage metric of the network at different values of $P_{f}$. We have noticed that the $k$ coverage probability of the network enhances with the increase in the number of SNs at a given value of $P_{f}$ as shown in Fig. 7, and Fig 8. For instance, the 1-coverage probabilities of the network are found to be 0.8766 , $0.9386,0.9694$ and 0.9848 when the number of SNs are 60, 80, 100 and 120 respectively at $P_{f}=0.6$ (refer Fig. 7). The reason behind this enhancement is that there exists a large number of SNs to cover any arbitrary square inside the rectangular RoI.Therefore, the number of squares covered increases with the increase in the number of SNs which in tern improves the $k$-coverage probability.

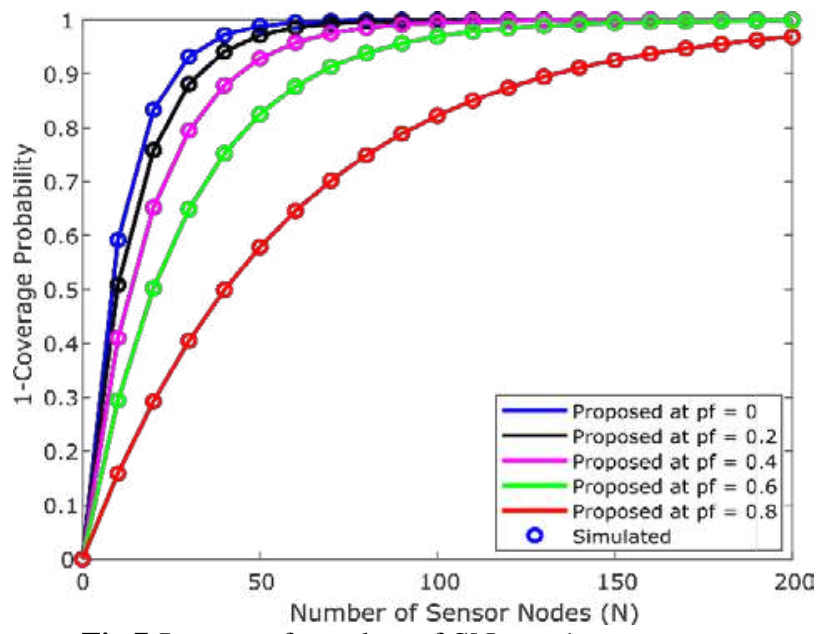

Fig.7:Impact of number of SNs on 1-coverage probability at different values of node failure rate $\left(P_{f}\right)$ and $r_{s}=80 \mathrm{~m}$

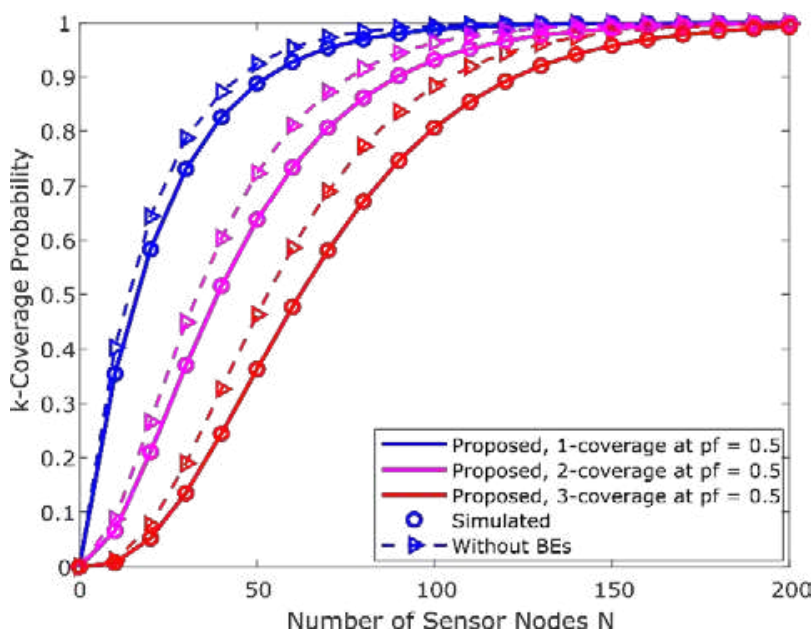

Fig.8:Impact of number of SNs on $k$-coverage probability when node failure rate $\left(P_{f}=0.5\right)$ and $r_{s}=80$ $\mathrm{m}$.

As discussed in the subsection 5.2, the $k$-coverage probability deteriorates with the increase in the value of sensor failure rate $\left(P_{f}\right)$. For instance, at $r_{s}=80 \mathrm{~m}$ and $N=$ 100, 1-coverage probabilities are found to be0.9998, $0.9992,0.9949,0.9694$ and 0.8224 when the values of $P_{f}$ are $0,0.2,0.4,0.6$ and 0.8 respectively as shown in Fig 7 . This is caused by the fact that at higher values of $P_{f}$, the expected number of failed nodes will be high, thus a less number of active SNs will available to cover entire region which in tern will decrease the 1-coverage probability.

Similarly, an increase in the value of $k$ will result in the decrease of $k$-coverage probability as shown in Fig. 6. For instance, at $r_{s}=80 \mathrm{~m}$ and $N=100,1$-coverage probabilities are found to be $0.9874,0.9313$ and 0.8069 for $k=1,2$ and 3 respectively (refer Fig. 8).The kcoverage probability decreases with the increase in the value of $k$ because a large value of $k$ requires that a given point inside the RoI must be covered by at least $k$ distinct SNs at a given value of $N$ and $r_{s}$. 


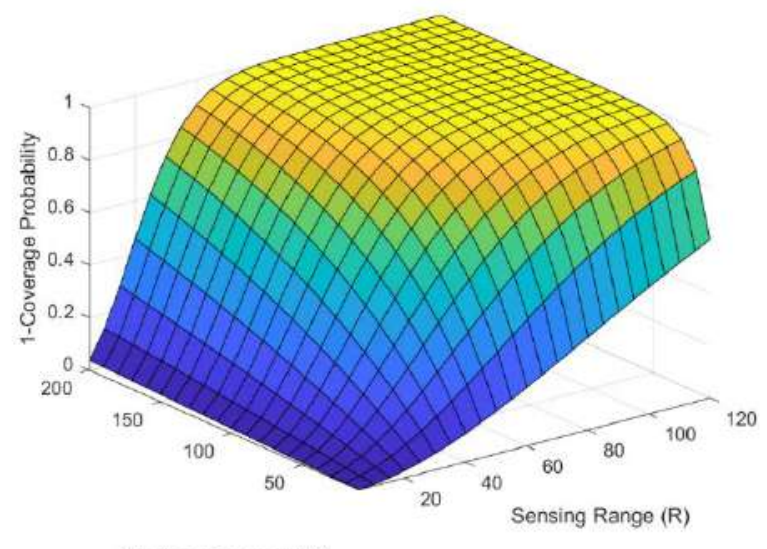

Number of sensors $(\mathrm{N})$

Fig.9:Three dimensional visualization of 1-coverage w.r.t. sensing range and number of SNs when $\left(P_{f}=0.5\right)$.

Here also, we found a major difference between the results with BEs and ignoring BEs as shown in Fig. 8. The simulation results match with the results obtained through the proposed model, thus validating our model. The simultaneous influence of both number of SNs and the sensing range on the 1-coverage probability of the network is shown in Fig. 9.

\section{Conclusion}

In this work, we have formulated an analytical model by considering BEs to study the influence of sensor failure rate $\left(P_{f}\right)$ and the network parameters such as number of SNs $\mathrm{N}$ and sensing range $\left(r_{s}\right)$ on the k-coverage probability of the network. The SNs with identical sensing range are assumed to spread uniformly and independently inside a rectangular RoI. It is observed that the k-coverage probability improves with the increase in the number of SNs and sensing range of SNs while deteriorates with the increase in the sensor failure rate $\left(P_{f}\right)$. The analytical results are validated through simulation results obtained through MATLAB 2018b and are found to be matching, thus confirming the proposed model. The proposed model will be useful for the researchers working in the area of WSNs as the model can be used to predict the coverage performance of the network before its actual deployment. In future work, we wish to examine the impact of various sensing range models along with the sensor failure rate on the k-coverage probability of the network.

As a part of future work, we can use various optimization $^{29,30)}$ and machine learning-based algorithms ${ }^{31,32,33,34)}$ to further improve the model performance.

\section{References}

1) Singh, Abhilash, Sandeep Sharma, and Jitendra Singh. "Nature-inspired algorithms for Wireless Sensor Networks: A comprehensive survey." Computer Science Review 39 (2021): 100342.

2) Amutha, J., Sandeep Sharma, and Sanjay Kumar
Sharma. "Strategies based on various aspects of clustering in wireless sensor networks using classical, optimization and machine learning techniques: Review, taxonomy, research findings, challenges and future directions." Computer Science Review 40 (2021): 100376.

3) Nagar, Jaiprakash, Sanjay K. Chaturvedi, and Sieteng Soh. "An analytical model to estimate the performance metrics of a finite multihop network deployed in a rectangular region." Journal of Network and Computer Applications 149 (2020): 102466.

4) Amutha, J., Sandeep Sharma, and Jaiprakash Nagar. "WSN strategies based on sensors, deployment, sensing models, coverage and energy efficiency: Review, approaches and open issues." Wireless Personal Communications 111, no. 2 (2020): 10891115.

5) Sharma, Sandeep, and Jaiprakash Nagar. "Intrusion Detection in Mobile Sensor Networks: A Case Study for Different Intrusion Paths." Wireless Personal Communications 115, no. 3 (2020): 2569-2589.

6) Kandris, Dionisis, Christos Nakas, Dimitrios Vomvas, and Grigorios Koulouras. "Applications of wireless sensor networks: an up-to-date survey." Applied System Innovation 3, no. 1 (2020): 14.

7) Nagar, Jaiprakash, and Sandeep Sharma. "k-Barrier coverage-based intrusion detection for wireless sensor networks." In Cyber Security, pp. 373-385. Springer, Singapore, 2018.

8) Tripathi, Abhishek, Hari Prabhat Gupta, Tanima Dutta, Rahul Mishra, K. K. Shukla, and Satyabrat Jit. "Coverage and connectivity in WSNs: A survey, research issues and challenges." IEEE Access 6 (2018): 26971-26992.

9) Singh, Abhilash, Sandeep Sharma, Jitendra Singh, and Rahul Kumar. "Mathematical modelling for reducing the sensing of redundant information in WSNs based on biologically inspired techniques." Journal of Intelligent \& Fuzzy Systems 37, no. 5 (2019): 6829-6839.

10) Mohamed, Shaimaa M., Haitham S. Hamza, and Iman Aly Saroit. "Coverage in mobile wireless sensor networks (M-WSN): A survey." Computer Communications 110 (2017): 133-150.

11) Liu, Ming, Jiannong Cao, Wei Lou, Li-jun Chen, and Xie Li. "Coverage analysis for wireless sensor networks." In International Conference on Mobile Ad-Hoc and Sensor Networks, pp. 711-720. Springer, Berlin, Heidelberg, 2005.

12) Laranjeira, Luiz A., and Genaína Nunes Rodrigues. "Border effect analysis for reliability assurance and continuous connectivity of wireless sensor networks in the presence of sensor failures." IEEE Transactions on Wireless Communications 13, no. 8 (2014): 4232-4246.

13) Nagar, Jaiprakash, Sanjay Kumar Chaturvedi, and Sieteng Soh. "Connectivity analysis of finite wireless 
multihop networks incorporating boundary effects in shadowing environments." IET Communications (2020).

14) Bettstetter, Christian. "On border effects in modeling and simulation of wireless ad hoc networks." In Proc. 3rd IEEE International Conference on Mobile and Wireless Communication Networks (WMAN), 2002. 2002.

15) Brust, Matthias R., Carlos HC Ribeiro, and José A. Barbosa Filho. "Border effects in the simulation of ad hoc and sensor networks." In 2009 11th International Conference on Computer Modelling and Simulation, pp. 180-185. IEEE, 2009.

16) Hechmi, Jridi Mohamed, Amira Zrelli, Mohamed Kbida, Hacen Khlaifi, and Tahar Ezzedine. "Coverage and Connectivity of WSN Models for Health Open-Pit Mines Monitoring." In 2018 14th International Wireless Communications \& Mobile Computing Conference (IWCMC), pp. 310-315. IEEE, 2018.

17) Amutha, J., Jaiprakash Nagar, and Sandeep Sharma. "A Distributed Border Surveillance (DBS) System for Rectangular and Circular Region of Interest with Wireless Sensor Networks in Shadowed Environments." Wireless Personal Communications (2020): 1-21.

18) Shen, Weiqiang, Chuanlin Zhang, and Jinglun Shi. "Weak k-barrier coverage problem in underwater wireless sensor networks." Mobile Networks and Applications 24, no. 5 (2019): 1526-1541.

19) Imran, Sajida, and Young-Bae Ko. "A continuous object boundary detection and tracking scheme for failure-prone sensor networks." Sensors 17, no. 2 (2017): 361.

20) Debnath, Sunandita, Ashraf Hossain, Sultan Mahmood Chowdhury, and Abhishek Kumar Singh. "Effective sensing radius (ESR) and performance analysis of static and mobile sensor networks." Telecommunication Systems 68, no. 1 (2018): 115-127.

21) Debnath, Sunandita, and Ashraf Hossain. "Network coverage in interference limited wireless sensor networks." Wireless Personal Communications 109, no. 1 (2019): 139-153.

22) Imani, Amir Hosein, Mohsen Eslami, Javad Haghighat, and Moslem Noori. "Effect of fading on the k-coverage of wireless sensor networks." Transactions on Emerging Telecommunications Technologies 31, no. 7 (2020): e3994.

23) Tarnaris, Konstantinos, Ioanna Preka, Dionisis Kandris, and Alex Alexandridis. "Coverage and kcoverage optimization in wireless sensor networks using computational intelligence methods: a comparative study." Electronics 9, no. 4 (2020): 675.

24) Singh, Abhilash, Vaibhav Kotiyal, Sandeep Sharma, Jaiprakash Nagar, and Cheng-Chi Lee. "A Machine
Learning Approach to Predict the Average Localization Error With Applications to Wireless Sensor Networks." IEEE Access 8 (2020): 208253208263.

25) Singh, Abhilash, Jaiprakash Nagar, Sandeep Sharma, and Vaibhav Kotiyal. "A Gaussian process regression approach to predict the k-barrier coverage probability for intrusion detection in wireless sensor networks." Expert Systems with Applications (2021): 114603.

26) Padmavathy, N., and Sanjay K. Chaturvedi. "Evaluation of mobile ad hoc network reliability using propagation-based link reliability model." Reliability Engineering \& System Safety 115 (2013): 1-9.

27) Khalid, Zubair, and Salman Durrani. "Distance distributions in regular polygons." IEEE Transactions on Vehicular Technology 62, no. 5 (2013): 2363-2368.

28) Khalid, Zubair, Salman Durrani, and Jing Guo. "A tractable framework for exact probability of node isolation and minimum node degree distribution in finite multihop networks." IEEE transactions on vehicular technology 63, no. 6 (2013): 2836-2847.

29) Kim, Young Deuk, Kyaw Thu, and Kim Choon Ng. "Evaluation and parametric optimization of the thermal performance and cost effectiveness of activeindirect solar hot water plants." Evergreen 2, no. 2 (2015): 50-60.

30) Alhamid, M., N. Nasruddin, E. Susanto, T. Vickary, and M. A. J. E. Budiyanto. "Refrigeration Cycle Exergy-Based Analysis of Hydrocarbon (R600a) Refrigerant for Optimization of Household Refrigerator." Evergreen 6, no. 1 (2019): 71-77.

31) Dief, Tarek N., and Shigeo Yoshida. "System Identification for Quad-rotor Parameters Using Neural Network." EVERGREEN Joint Journal of Novel Carbon Resource Sciences \& Green Asia Strategy 3, no. 01 (2016): 6-11.

32) Aliunir, Fachransjah, Teuku Yuri M. Zagloel, and Romadhani Ardi. "Discrete-Event Simulation and Optimization of Spare Parts Inventory and Preventive Maintenance Integration Model Considering Cooling Down and Machine Dismantling Time Factor." EVERGREEN Joint Journal of Novel Carbon Resource Sciences \& Green Asia Strategy 7 (2020): 79-85.

33) Choudhary, Shilpa, Abhishek Sharma, Shradha Gupta, Hemant Purohit, and Smriti Sachan. "Use of RSM Technology for the Optimization of Received Signal Strength for LTE Signals Under the Influence of Varying Atmospheric Conditions."EVERGREEN Joint Journal of Novel Carbon Resource Sciences \& Green Asia Strategy (2020): 500-509.

34) Kotiyal, Vaibhav, Abhilash Singh, Sandeep Sharma, Jaiprakash Nagar, and Cheng-Chi Lee. "ECS-NL: An Enhanced Cuckoo Search Algorithm for Node 


\section{Localisation in Wireless Sensor}

Networks." Sensors 21, no. 11 (2021): 3576. 